\title{
miR172 Regulates Soybean Nodulation
}

\author{
Zhe Yan, ${ }^{1}$ Md Shakhawat Hossain, ${ }^{1}$ Jun Wang, ${ }^{1,2}$ Oswaldo Valdés-López, ${ }^{1}$ Yan Liang, ${ }^{1}$ Marc Libault, ${ }^{1}$ \\ Lijuan Qiu, ${ }^{2}$ and Gary Stacey ${ }^{1}$ \\ ${ }^{1}$ Divisions of Plant Science and Biochemistry,, National Center for Soybean Biotechnology, Christopher S. Bond Life \\ Sciences Center, University of Missouri, Columbia, MO 65211, U.S.A.; ${ }^{2}$ National Key Facility for Crop Gene Resources and \\ Genetic Improvement (NFCRI), Institute of Crop Science, Chinese Academy of Agricultural Sciences, Beijing 100081, China
}

Submitted 22 April 2013. Accepted 20 August 2013.

\begin{abstract}
Micro-RNAs (miRNAs) play a pivotal role in the control of gene expression and regulate plant developmental processes. miRNA 172 (miR172) is a conserved miRNA in plants reported to control the expression of genes involved in developmental phase transition, floral organ identity, and flowering time. However, the specific role of miR172 in legume nodulation is undefined. Ectopic expression of soybean miR172 resulted in an increase in nodule numbers in transgenic roots and an increase in the expression of both symbiotic leghemoglobin and nonsymbiotic hemoglobin. These nodules showed higher levels of nitrogenase activity. Further analysis revealed a complex regulatory circuit in which miR156 regulates miR172 expression and controls the level of an AP2 transcription factor. The latter, either directly or indirectly, controls the expression of nonsymbiotic hemoglobin, which is essential for regulating the levels of nodulation.
\end{abstract}

The rhizobium-legume nitrogen-fixing symbiosis is agronomically and ecologically important. This symbiosis results in the formation of a new root organ, the nodule, in which the rhizobia establish an intracellular symbiosis (Murray 2011). Rhizobial infection of legumes occurs primarily through root hairs, single-cell extensions of the root epidermis. The rhizobia infect the root hairs and gain access to the nodule cells through a tubular infection thread, specifically induced in response to rhizobial signals. The infecting rhizobia induce cortical cell divisions leading to nodule development. The rhizobia are ultimately released from the infection threads through an endocytotic process and colonize cells within a membrane-bound vacuolar-like compartment, termed a symbiosome (Roth et al. 1988). The symbiosome is the functional unit of biological nitrogen fixation (Kereszt et al. 2011).

Rhizobia fix atmospheric nitrogen via the nitrogenase enzyme (Kim and Rees 1994). Although cleavage of the $\mathrm{N}_{2}$ triple bond is exergonic, considerable activation energy is required that is provided by $16 \mathrm{~mol}$ of ATP per $\mathrm{N}_{2}$ reduced (Ljones and

Zhe Yan, Md Shakhawat Hossain, and Jun Wang contributed equally to this work.

Corresponding author: G. Stacey; Telephone: +01-5738844752; Fax: +015738849676; E-mail: staceyg@ missouri.edu

* The $e$-Xtra logo stands for "electronic extra" and indicates that three supplementary figures and one supplementary table are published online.

(C) 2013 The American Phytopathological Society
Burris 1972). The nitrogenase enzyme is extremely oxygen labile and, hence, needs to be protected from free oxygen. However, rhizobia are obligate aerobes. In the nodule, the rhizobia induce the expression of high-affinity, terminal oxidases, while the plant produces leghemoglobin ( $\mathrm{Lb}$ ) in high amount, which effectively binds all free oxygen. These adaptations allow the rhizobia to fix nitrogen aerobically in an environment in which nitrogenase is protected (Cutting and Schulman 1971; Wittenberg et al. 1972).

In legumes, there are two main types of hemoglobin, including symbiotic $\mathrm{Lb}$ and nonsymbiotic hemoglobin $(\mathrm{Hb})$ (Anderson et al. 1996). Hb was originally found in the only nonleguminous plant known to be nodulated by rhizobia, a Parasponia sp. (Ulmaceae) (Appleby et al. 1983). Later, Hb was identified in a large variety of nonnodulated plants (Hardison 1996). Hb was also identified to function in nodulation (Shimoda et al. 2009). In Lotus spp. ectopic expression of $\mathrm{Hb}$ was found to increase nodule numbers with a concomitant increase in nitrogenase activity (Shimoda et al. 2009).

In recent years, many studies demonstrated an important role for micro-RNA (miRNAs) during plant development (Rubio-Somoza and Weigel 2011; Zhang et al. 2006). For instance, miR172 is a conserved miRNA in plants, present in all angiosperm lineages. In Arabidopsis, miR172 associates with AGO1 or AGO10 (Ji et al. 2011) and acts to silence specific target gene expression. For example, Arabidopsis miR172 targets the transcription factor gene APETALA2 $(A P 2)$, which regulates juvenile to adult-phase transition, floral organ identity, and flowering time (Aukerman and Sakai 2003; Chen 2004; Lauter et al. 2005; Nair et al. 2010). Arabidopsis miR156 is a negative regulator of miR172 expression (Wu et al. 2009). miR156 targets SPL transcription factors, which directly bind to the miR 172 promoter and positively regulate miR172 expression. In legumes, miR172 accumulates to high levels in mature nodules, including those formed on Medicago truncatula, Lotus japonicus, common bean, and soybean, relative to the levels in other tissues, such as leaves and roots (De Luis et al. 2012; Lelandais-Briere et al. 2009; Valdes-Lopez et al. 2010; Wang et al. 2009). However, a direct, functional role for miR172 in nodulation has not demonstrated.

Here, we report data from soybean suggesting a complex regulatory circuit in which the levels of miR156 negatively control miR172 expression, which targets the mRNA of the AP2-2 transcription factor. The latter, either directly or indirectly, regulates the expression of $\mathrm{Hb}$, which is essential for regulating the levels of nodulation. The net effect is that the level of nodulation is controlled by the interaction of miR 156 and miR172, which impacts the expression of their downstream target genes. 


\section{RESULTS}

\section{miR172 regulates soybean nodulation.}

Previous studies demonstrated that the level of miR172 accumulated in mature nodules in different legume species, including soybean (De Luis et al. 2012; Lelandais-Briere et al. 2009; Valdes-Lopez et al. 2010; Wang et al. 2009). In order to examine the expression activity of miR172 in soybean nodule development, we measured the levels of miR172 in different stages of soybean nodules using quantitative reverse-transcriptase polymerase chain reaction (qRT-PCR). We selected soybean nodules according to their relative symbiotic nitrogen-fixation activity (Pessi et al. 2007) and harvested them 10, 20, and 30 days after rhizobial inoculation (DAI). At 10 DAI, the nodules can be clearly distinguished but are still developing; at approximately 20 DAI, the relative symbiotic nitrogen fixation activities, under our growth conditions, were the highest and, therefore, representing the mature, fully functioning nodules; at approximately 30 DAI, the relative symbiotic nitrogen fixation activities decrease to $61 \%$, representing nodules that have already begun to senescent. Compared with 10-DAI nodules, the expression of miR172 was increased significantly 20 DAI. However, miR172 expression was slightly decreased at 30 DAI (Fig. 1).

In order to investigate the role of miR172 during soybean nodulation, the miR172 precursor gene was ectopically expressed from the strong Cassava mosaic virus (CvMV) promoter in the binary vector pCAMGFP-CvMV:GWOX, which expresses a constitutive green fluorescent protein (GFP) cassette (Li et al. 2010). Ectopic expression of miR172 resulted in a 2.4-fold increase in nodule numbers (Fig. 2). The qRT-PCR analysis showed that the levels of miR172 were increased 9.3fold by expression from the CvMV promoter, relative to transgenic roots transformed with the empty vector (Fig. 3).

\section{miR172 expression affects nitrogenase activity and hemoglobin accumulation.}

Given the strong effects of miR172 expression on nodule formation, we also measured nodule nitrogenase activity using the acetylene-reduction assay. Compared with the empty vector control, ectopic expression of miR172 resulted in a significant increase in nitrogenase activity (Fig. 4). According to previous reports, the levels of both symbiotic hemoglobin and $\mathrm{Hb}$ can

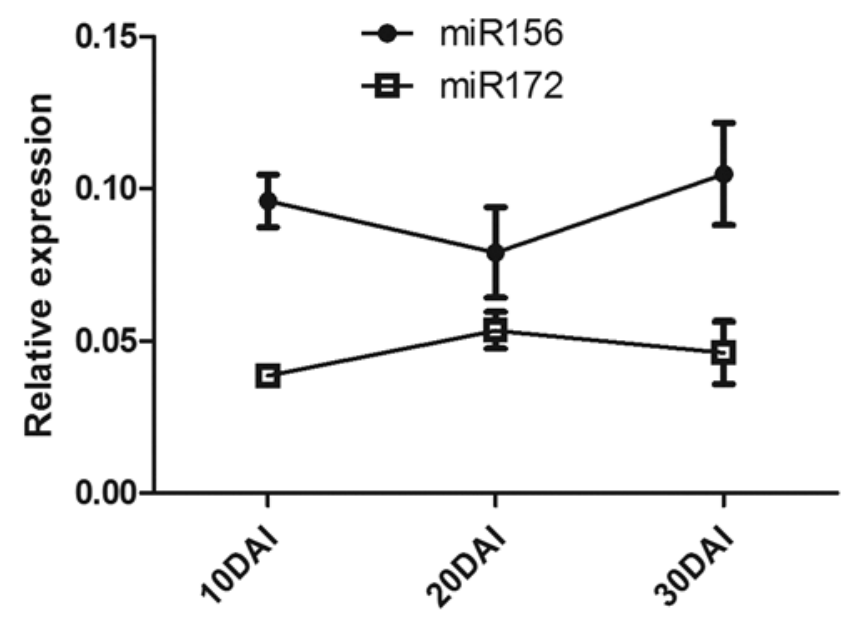

Fig. 1. Relative expression level of micro-RNA 172 (miR172) and miR156 between 10 to 30 days after Bradyrhizobium japonicum inoculation (DAI). The expression of miR156 and miR172 was measured by quantitative reverse-transcriptase polymerase chain reaction 10, 20, and 30 DAI nodules. Three biological replications were performed. The relative expressions of miR156 and miR172 were calculated by the expression of reference genes snoR 1 and 5.8S. Error bar indicates \pm standard error. affect nitrogenase activity (Dakora 1995; Shimoda et al. 2009). A sequence-based search of the soybean genome sequence identified six hemoglobin genes, including four Lb genes ( $L b a$, $L b c 1, L b c 2$, and $L b c 3)$ and two $\mathrm{Hb}$ genes ( $\mathrm{Hb}-\mathrm{l}$ and $\mathrm{Hb}-2)$. We designed specific primers for each of these genes and used them to measure gene expression by qRT-PCR. In the CvMV:: miR172 tissues, mRNA levels of one $L b$ gene, Glyma10g34280 $(L b c 1)$, and the two $H b$ genes, Glyma11g12960 $(H b-1)$ and Glyma11g12980 ( $\mathrm{Hb}-2)$, were significantly increased compared with the empty vector control (Fig. 5). There were no significant changes in the expression levels of $L b a, L b c 2$, and $L b c 3$ in the CvMV::miR172 tissues (data not shown).

\section{An AP2 transcription factor regulates nodulation.}

According to previous reports, miR172 targets the plant-specific transcription factor gene $A P 2$ in different plant species (Aukerman and Sakai 2003). According to target prediction (Joshi et al. 2010), we predicted 11 AP2 genes as targets for soybean miR172. Target gene qRT-PCR analysis revealed that the levels of 3 of these 11 AP2 transcription factors were decreased significantly in CvMV::miR172 tissues (Fig. 3), including Glyma03g33470 (AP2-1), Glyma11g15650 (AP2-2), and Glyma19g36200 (AP2-3). The other eight AP2 expression levels were not affected by miR172 (data not shown). In order to investigate the possible role of these $A P 2$ genes in nodulation, we designed specific RNAi silencing constructs for each $A P 2$ gene and expressed these in transgenic soybean roots (Fig. 2A). Compared with transgenic roots expressing a $\beta$-glucuronidase-RNAi construct, there was no significant change in nodule numbers in roots expressing either $A P 2-1$ RNAi or AP2-3 RNAi. However, expression of the AP2-2 RNAi resulted in a significant increase in nodule numbers (Fig. 2A), with no effect on the expression of AP2-1 and AP2-3 (Supplementary Fig. S1).

\section{$A P 2-2$ represses nodulation and negatively regulates $H b-1$ accumulation in soybean.}

Given the results above, we focused specifically on the role of AP2-2 in nodulation. We constructed an AP2-2 overexpression vector and transformed soybean roots. A significant reduction in nodule formation was seen in roots ectopically expressing AP22 relative to transgenic roots transformed with the empty vector (Fig. 2A and B). Given the results that miR172 affects hemoglobin levels, we also measured hemoglobin levels in nodules formed on the CvMV::AP2-2 roots. Ectopic expression of $A P 2-2$ significantly decreased $H b-1$ expression levels (Fig. 6). Contrary to the results found with roots transformed with the CvMV::miR172 construct, there were no apparent differences in the levels of $L b c l$ and $H b$-2 expression in the AP2-2-expressing roots. We assume that the effects on $L b c l$ and $H b-2$ expression are likely mediated by another, unknown transcription factor that may also be targeted by miR172. We also measured $H b-1$ expression levels in AP2-2 RNAi tissues. Consistent with the results obtained by ectopic expression of $A P 2-2$, RNAi silencing of $A P 2-2$ expression resulted in increased expression of $\mathrm{Hb}-\mathrm{l}$. However, given the variation in these experiments, the effect was not statistically significant (Supplementary Fig. S2).

Interestingly, in CvMV::AP2-2 transgenic tissues, the expression of miR172 was decreased significantly compared with the control. However, the expression of miR156 remained unaffected. Therefore, although the levels of miR172 and miR156 showed a negative correlation in CvMV::AP2-2 transgenic tissues, the data would suggest that $A P 2-2$ does not suppress miR172 expression by elevating levels of miR156 (Fig. 6).

Ecotopic expression of $\boldsymbol{H} \boldsymbol{b}$ - $\boldsymbol{1}$ enhances soybean nodulation.

Previously, Shimoda and associates (2009) reported that ectopic expression of $\mathrm{Hb}$ in $L$. japonicas roots resulted in a 
significant increase in nodule numbers upon rhizobial inoculation and a strong enhancement of nodule nitrogenase activity (Shimoda et al. 2009). These results suggested the possibility that the increase in nodule formation seen on roots ectopically expressing miR 172 could be due to a reduction in $A P 2$ expression, thereby relieving the repressive effect of this transcription factor on $\mathrm{Hb}-\mathrm{I}$ expression (Fig. 5). In order to test this hypothesis, we transformed soybean transgenic roots with a CvMV::Hb-1 construct and compared nodulation with roots transformed with the empty vector. Consistent with the hypothesis, those roots expressing $\mathrm{Hb}-\mathrm{l}$ produced significantly more nodules than the control roots (Fig. 2A and B). We also measured the nitrogenase activity of nodules formed on the CvMV:: $H b-1$ roots. As expected, the CvMV::Hb-1 roots showed significantly higher nitrogenase activity (Fig. 4).

\section{miR156 is a negative regulator of miR172 expression during soybean nodulation.}

According to a previous study in Arabidopsis, miR156 represses miR172 expression by targeting an SPL transcription factor, which is required for transcription of the miR172 premRNA (Wu et al. 2009). Therefore, we also measured the expression of soybean miR156 in nodules formed 10, 20, and 30 DAI. The expression of miR156 was negatively correlated with miR172 levels at all time points measured (Fig. 1).

We also analyzed the nodulation phenotype of soybean transgenic roots transformed with a CvMV::miR156 construct. These roots showed a marked reduction in nodulation $(40 \%$ reduction) compared with roots transformed with the empty vector (Fig. 2). These results are consistent with the notion that, similar to Arabidopsis, miR156 represses expression of miR172. Consistent with this hypothesis, the expression of miR172 was decreased by $44 \%$ in the CvMV::miR156-transformed roots. We also measured the expression of the predicted mRNA targets of miR156, which were predicted in our previous article (Joshi et al. 2010). miRNA156 targets SPL transcription factors. Indeed, the mRNA levels of two SPL transcription factors, Glyma08g01450 (SPL 1) and Glyma12g27330 (SPL 2), were significantly decreased in the CvMV::miR156transformed roots (Fig. 7). We also observed miR172 targets (AP 2-1, AP 2-2, and $A P$ 2-3) and $H b l$ expression in the CvMV::miR156-transformed tissues. As expected, we found that miR172 target expression was increased significantly, while $\mathrm{Hb} 1$ decreased (Fig. 7). Moreover, the CvMV::miR156

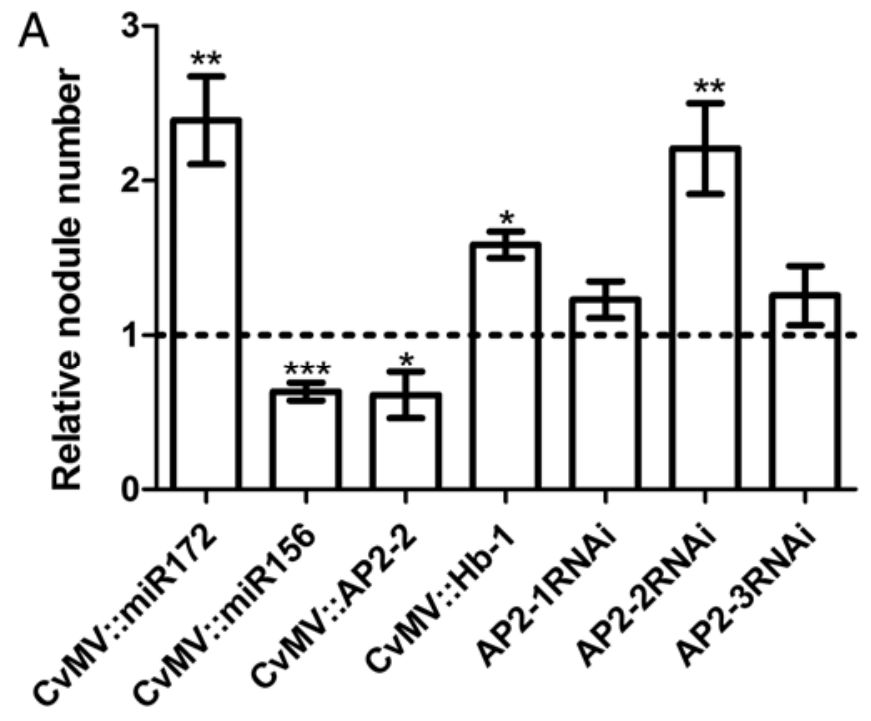

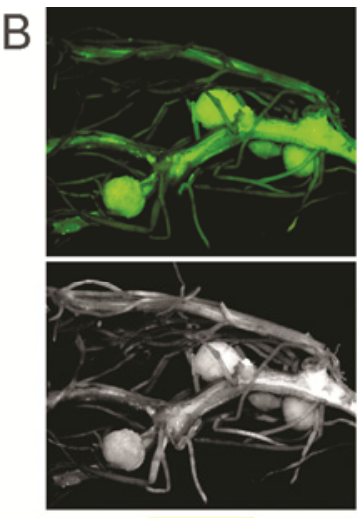

Control

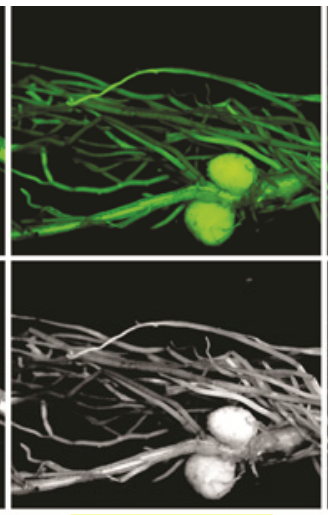

CvMV::miR156

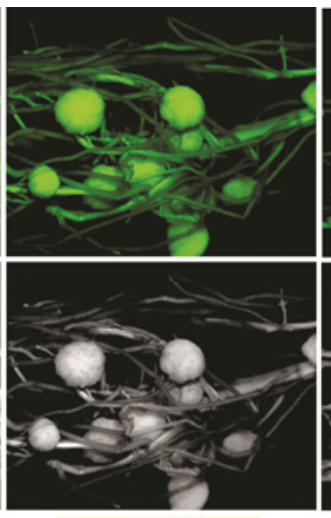

CvMV::miR172

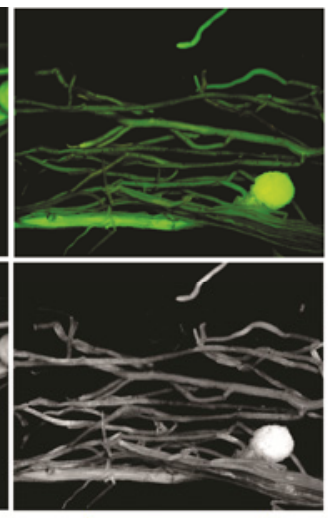

CvMV::AP2-2

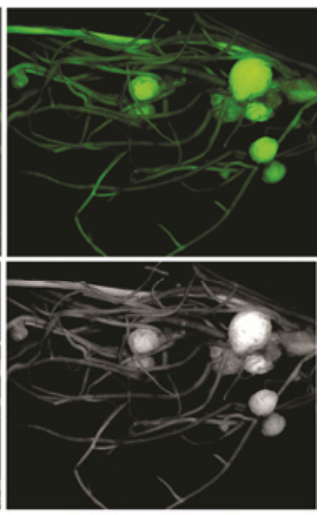

CvMV::Hb-1

Fig. 2. Soybean nodule numbers were affected by micro-RNA 172 (miR172), miR156, $A P 2-2$, and $H b-1$. A, Relative nodule numbers formed on soybean transgenic roots either ectopically expressing miR172, miR156, $A P 2-2$, and Hb-1 or in which the expression of the $A P 2-1$, $A P 2-2$, or $A P 2-3$ genes was silenced using RNAi. Nodule numbers were counted 6 weeks after inoculation. Relative nodule numbers formed on soybean transgenic hairy roots were calculated by the ratio change between treatments with control roots, which were transformed with the empty vector. Error bar indicates \pm standard error. The $t$ test was performed using the null hypothesis that the fold-change difference between the experimental and control was equal to one; $*, * *$, and $* * *$ indicate significance at the $0.05,0.01$, and 0.005 probability level, respectively. B, Images of soybean transgenic roots ectopically expressing miR 156 , miR $172, A P 2$ 2, or $\mathrm{Hb}-1$ from the strong CvMV promoter. Representative photos showing nodulation. Top: fluorescent image showing transgenic expression of green fluorescent protein (GFP). Bottom: corresponding light micrographs. Fluorescence arises from constitutive expression of GFP in Agrobacterium rhizogenes transformed roots. Control: transformed with empty vector pCAMGFP-CvMV-GWOX. 
roots also showed a significant decrease in nitrogenase activity (Fig. 4). The ectopic expression of either miR156 or miR172 did not affect nodule morphology (Supplementary Fig. S3). Collectively, the results suggest a model in which miR156 negatively regulates miR172 expression, thereby controlling hemoglobin expression, nitrogenase activity, and soybean nodule number.

\section{DISCUSSION}

miRNAs play pivotal roles in the control of gene expression and regulate plant developmental processes. mR172 is a conserved miRNA in plants. Levels of miR172 were shown to

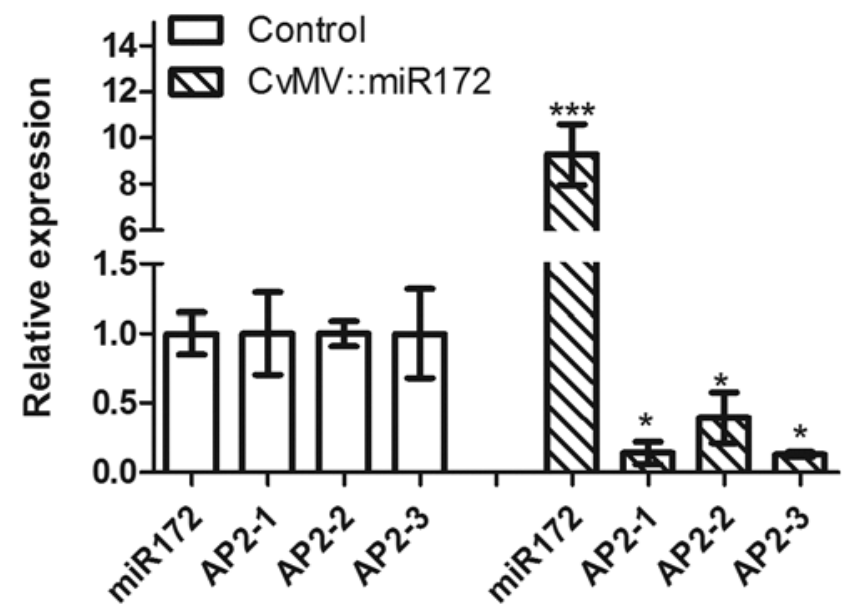

Fig. 3. Relative expression levels of micro-RNA 172 (miR172) and its predicted target genes in soybean transgenic roots ectopically expressing miR172 from the strong CvMV promoter. The expression level of miR172 and $A P 2-1, A P 2-2$, and $A P 2-3$ were measured in CvMV::miR172 transgenic tissues. The relative expression was calculated by dividing the experimental value and the control value. Three replications were performed. Error bar indicates \pm standard error; * and $* * *$ indicate significance at the 0.05 and 0.005 probability level, respectively.

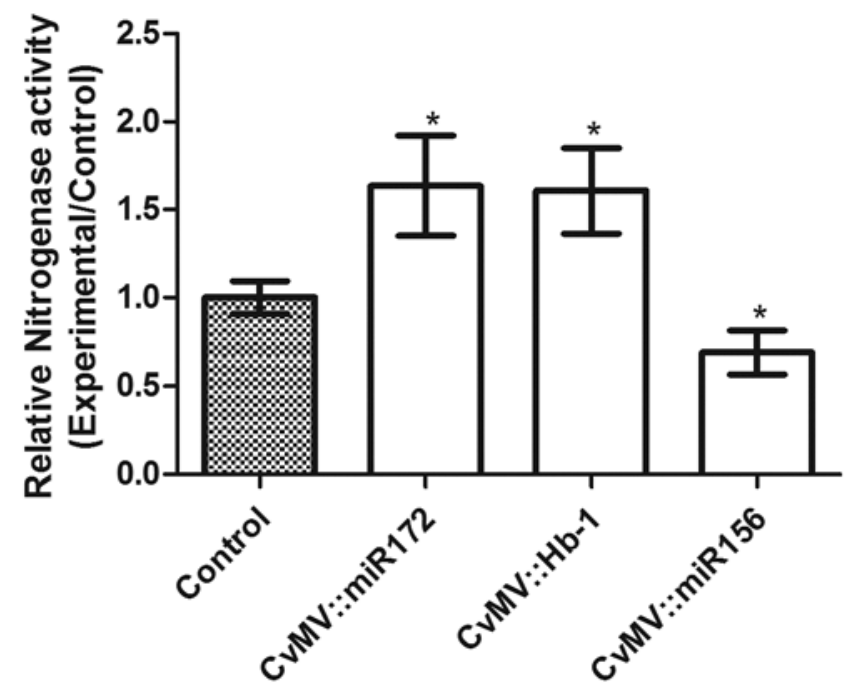

Fig. 4. Relative nitrogenase activity of nodules formed on transgenic roots expressing micro-RNA 172 (miR172), Hb-1, or miR156 from the strong CvMV promoter. Nodule nitrogenase activity on roots expressing miR172, CvMV::HB-1, or miR156 was measured using the acetylene reduction assay and compared with roots transformed with the empty vector. Nodules were harvested 6 weeks after inoculation. The relative nitrogenase activity was calculated by dividing the experimental value and the control value. Two replications were performed. Error bar indicates \pm standard error; * indicates significance at the 0.05 probability level. affect Arabidopsis growth-phase transition and flower development (Aukerman et al. 2003; Chen 2004; Lauter et al. 2005; Nair et al. 2010) and the expression of miR172 promotes Arabidopsis adult identity (Wu et al. 2009). The data presented here indicate that soybean miR172 is a regulator of nodule development. Transgenic soybean roots ectopically expressing miR172 from the strong, constitutive CvMV promoter produced significantly more nodules than transgenic roots transformed with the empty vector. Further analysis showed that these transgenic roots also showed an increase in the expression of both symbiotic $\mathrm{Lb}$ and $\mathrm{Hb}$. The nodules of CvMV:: miR172 also showed higher levels of nitrogenase activity. Comparing miR172 expression in nodules in different development stages, miR172 levels were higher at 20 DAI compared with 10 and 30 DAI. As previously reported (Pessi et al. 2007), nodules at 10, 20, and 30 DAI exhibit 9, 100, and $61 \%$

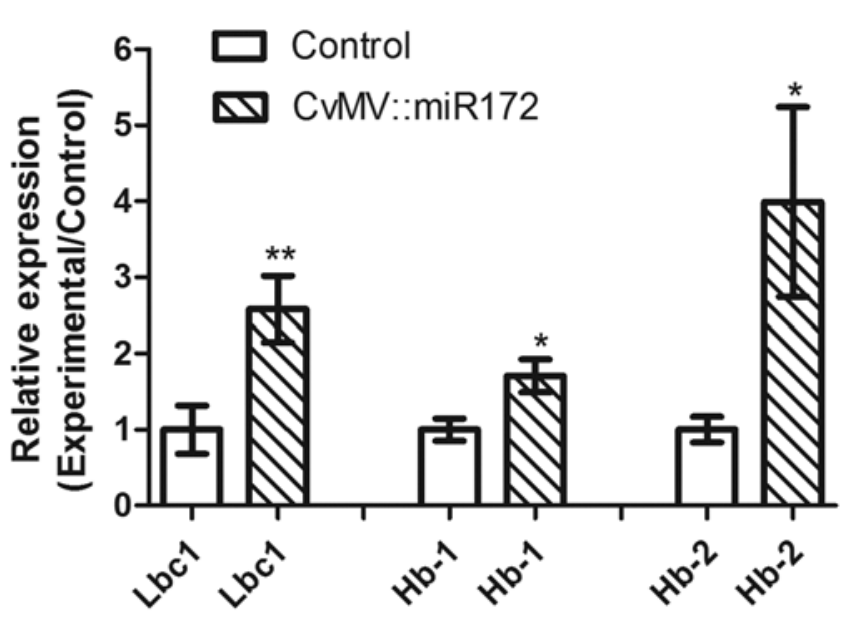

Fig. 5. Relative expression of $L b c 1, H b-1$, and $H b-2$ in transgenic roots expressing micro-RNA 172 (miR172) from the strong CvMV promoter. Expression levels of $L b c 1, H b-1$, and $H b-2$ were measured in CvMV:: miR172 transgenic tissues. The relative expression was calculated by dividing the experimental value and the control value. Three replications were measured. Error bar indicates \pm standard error; $*$ and $* *$ indicate significance at the 0.05 and 0.01 probability level, respectively.

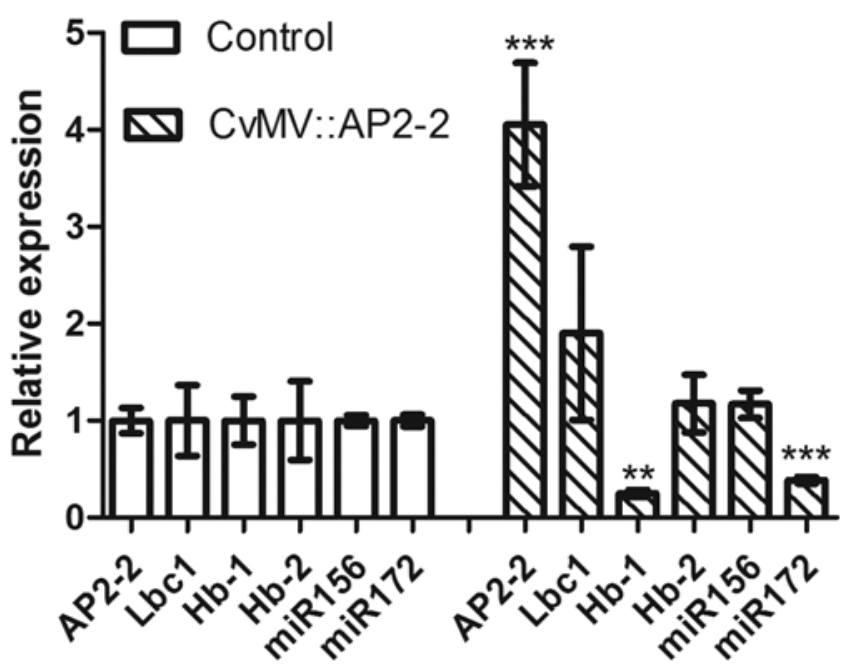

Fig. 6. Relative expression of hemoglobin, micro-RNA 156 (miR156), and miR172 in CvMV::AP2-2 transgenic nodules. Expression levels of AP2-2, hemoglobin genes ( $L b c l, H b-1$, and $H b-2)$, miR156, and miR172 were compared between control plants transformed with empty vector and CvMV:: AP2-2 transgenic tissues. The relative expression was calculated by dividing the experimental value and the control value. Three replications were measured. Error bar indicates \pm standard error; $* *$ and $* * *$ indicate significance at the 0.01 and 0.005 probability level, respectively. 
relative nitrogen fixation (i.e., acetylene reduction) activities, respectively. These levels are roughly consistent with the miR172 expression pattern in nodules, suggesting either a direct or indirect role for miR172 in controlling nodule nitrogenase activity.

Symbiotic Lb facilitates oxygen transport to rhizobia in nodules to support energy production, while also limiting free oxygen concentration in the nodules to protect the oxygen-sensitive nitrogenase enzyme (O'Brian et al. 1987). In L. japonicus, the expression of $\mathrm{Hb}$ increased nitrogenase activity and improved nodulation (Shimoda et al. 2009). In this study, we showed that, similar to the studies in L. japonicus, strong ectopic expression of $\mathrm{Hb}$ in soybean transgenic roots significantly increased nodule numbers and nitrogenase activity but without obvious morphological differences.

AP2 transcription factors are involved in a variety of plant processes, including phase transition (Lauter et al. 2005), floral organ identity (Chen 2004), cleistogamy (Nair et al. 2010), flowering time (Aukerman and Sakai 2003), stem cell niche (Wurschum et al. 2006), seed mass (Ohto et al. 2005), and plant resistance to necrotrophic pathogens (Zhao et al. 2012). For example, AP2 members, such as AP2-like ethylene-responsive transcription factor $(S M Z)$, act as repressors of flowering during Arabidopsis development. Distinct SMZ binding sites were identified in the promoters of FLOWERING LOCUS T (FT). SMZ binding to these sites represses $F T$ expression and, therefore, strongly affects flowering (Mathieu et al. 2009).

In soybean, the data indicate that miR172 targets an AP2-2 transcription factor, which either directly or indirectly represses $\mathrm{Hb}$ gene expression. This is consistent with previous reports showing that CPP1, a cysteine-rich repeat protein, is induced during nodulation and binds to the $L b c 3$ promoter and represses $L b c 3$ expression (Cvitanich et al. 2000). ChIP-seq analysis of the Arabidopsis genome identified thousands of AP2-binding loci, including an Arabidopsis hemoglobin gene (Yant et al. 2010). Although the ability of AP2-2 to directly bind to the $H b-1$ promoter was not tested, the data presented argue that $A P 2-2$ does play a role in repression of $H b-1$ expression.

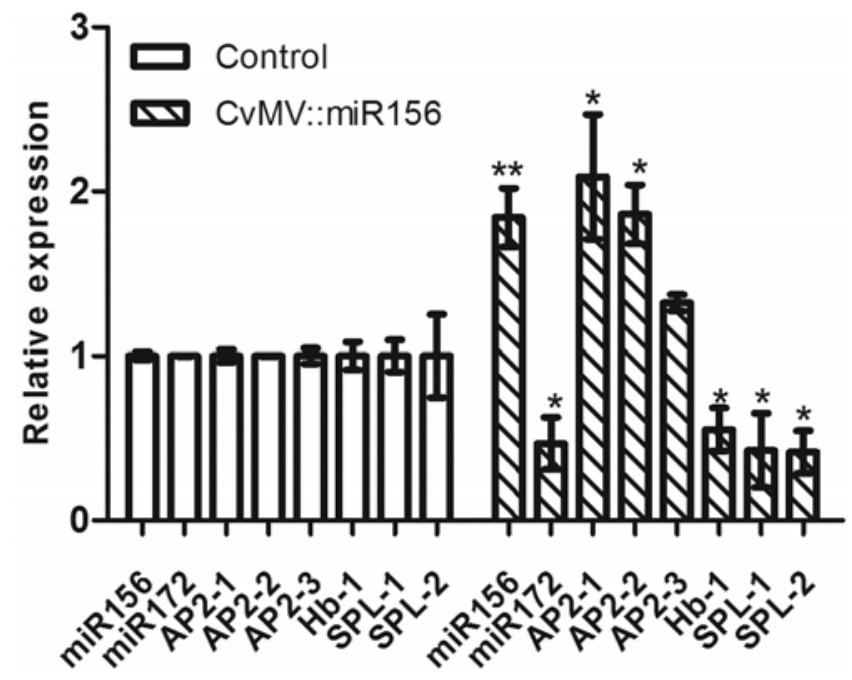

Fig. 7. Relative expression of micro-RNA 156 (miR156), miR172, AP2, and $\mathrm{Hb}$-lgenes in nodules ectopically expressing miR156. Expression levels of miR156, miR172, AP2-1, AP2-2, AP2-3, and $H b-1$ were measured by quantitative reverse-transcriptase polymerase chain reaction in CvMV:: miR156 transgenic tissues and compared with roots transformed with the empty vector. The relative expression was calculated by dividing the experimental value and the control value. Three replications were measured. Error bar indicates \pm standard error; $*$ and $* *$ indicate significance at the 0.05 and 0.01 probability level, respectively.
Previous research in Arabidopsis showed that miR156 is a negative regulator of miR172 expression (Wu et al. 2009). miR156 targets SPL transcription factors, which directly bind to the miR172 gene promoter and positively regulate miR172 expression. In addition to Arabidopsis, miR156 was also shown to negatively regulate miR172 expression in maize (Chuck et al. 2007) and Populus $\times$ canadensis (Wang et al. 2011). In both Arabidopsis and maize, miR156 and miR172 play a role in developmental phase transition. In the case of soybean nodulation, the data are also consistent with a role for miR156 in the negative regulation of miR172 expression. This was seen in transgenic plants ectopically expressing miR156, which resulted in reduction in miR172 levels, with a concomitant increase in AP2-2 expression and a reduction in nodule formation. We also found a strong negative correlation between miR156 and miR172 levels during a time course of nodule formation (10 to 30 DAI) (Fig. 1).

The results presented, when taken as a whole, suggest the model shown in Figure 8. This postulated regulatory circuit suggests that soybean nodule formation is controlled by the opposing effects of miR156 and miR172 accumulation. miR156 represses the expression of its targets, SPL transcription factors, which regulate miR172 expression; miR172 controls AP2-2 expression and directly or indirectly affects $\mathrm{Hb}-1$ levels. The level of $H b-1$ positively affects soybean nodulation. What is not clear and will require further research is how $\mathrm{Hb}-1$ levels are regulated and how $H b-1$, either directly or indirectly, controls the number of nodules formed and their relative nitrogenase activity.

\section{MATERIALS AND METHODS}

Plant materials.

Methods for plant culture were modified from Malik and associates (1987). Soybean seed were surface sterilized with $30 \%$ bleach, rinsed several times with autoclaved double-distillated $\mathrm{H}_{2} \mathrm{O}\left(\right.$ ddiH $\left._{2} \mathrm{O}\right)$, once with $0.01 \mathrm{M} \mathrm{HCl}$ for $10 \mathrm{~min}$, and then several times with autoclaved $\operatorname{ddiH}_{2} \mathrm{O}$. Sterilized seed were planted on plates contained nitrogen-free Broughton and Dilworth agar medium (Broughton and Dilworth 1971) and germinated in a growth chamber in the dark at $27^{\circ} \mathrm{C}$ and $80 \%$ humidity. After 3 days, soybean seedlings were transferred to plastic growth pouches. The paper wick within each plant pouch was wetted with nitrogen-free plant nutrient solution (Subramanian et al. 2004). Seedlings were grown in an environmentally controlled chamber maintained at a 14-h photoperiod with temperature maintained at $28^{\circ} \mathrm{C}$ during the day and $26^{\circ} \mathrm{C}$ during the night. Soybean roots were inoculated with Bradyrhizobium japonicum USDA110. Nodules were collected 10, 20, and 30 DAI.

\section{Total RNA isolation and RT-PCR.}

Total RNA was purified using Trizol Reagent according to the manufacturer's instructions (Invitrogen, Carlsbad, CA, U.S.A.) and subsequently purified using chloroform extraction.

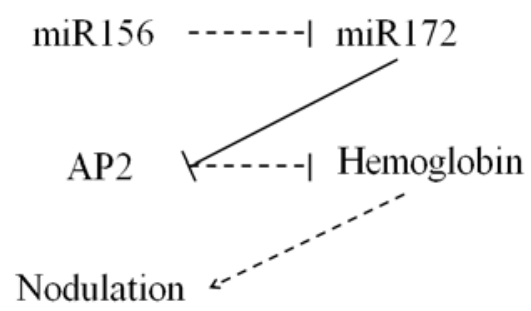

Fig. 8. Model of micro-RNA 156 (miR156) and miR172 regulation of soybean nodulation. 
Genomic DNA (gDNA)-free RNA $(1 \mu \mathrm{g})$ was reverse transcribed using Superscript III (Invitrogen). Specific primers were designed for each candidate gene (Supplementary Table S1C) and qRT-PCR was performed as described by Libault and associates (2008). Con4 and Con15 were used as reference genes to normalize gene expression.

\section{MicroRNA qRT-PCR analysis.}

Quantification of mature miRNA abundance was performed by qRT-PCR following the protocol described by VarkonyiGasic and associates (2007). gDNA-free RNA (1 $\mu \mathrm{g})$ was reverse transcribed using Superscript III (Invitrogen) using a stem-loop-specific RT primer, which contained six nucleotidespecific sequences from the complementary $3^{\prime}$ end sequence of each miRNA. qRT-PCR was performed using SYBR Green PCR mix (ABI enzyme) using an miRNA-specific forward primer and a universal reverse primer (Varkonyi-Gasic et al. 2007). The reference genes snoR 1 and $5.8 \mathrm{~S}$ were selected to calculate the relative expression of each miRNA.

\section{Plasmid construction \\ and soybean hairy root transformation.}

Agrobacterium rhizogenes-dependent hairy root transformation was used to construct transgenic soybean roots ectopically expressing the selected miRNAs. The vector pCAMGFPCvMV-GWOX (Li et al. 2010) was used, which allowed cloning of the precursor mRNA of each of the miRNA behind the strong CvMV promoter. The T-DNA of this vector also contains a constitutively expressed GFP cassette, which enables identification of transformed roots by GFP fluorescence (Li et al. 2010). The nodulation phenotypes of only the transformed roots were measured and compared with empty vector controls.

To clone the miRNA precursors, miR172 $\mathrm{j}$ and miR156o were selected. The regions of DNA spanning 200 bp upstream and downstream of each mature miRNA region were amplified from soybean gDNA using specific primers. The amplified precursors were cloned in the pDONR-Zeo vector (Invitrogen) and sequenced. For $A P 2-2$ and $H b-1$ overexpression cloning, the fragments containing the coding region of $A P 2-2$ or $\mathrm{Hb}-\mathrm{I}$ was amplified by PCR and cloned in the pDONR-Zeo vector. The resulting plasmids were recombined into the pCAMGFPCvMV-GWOX binary vector using the Gateway LR Clonase II enzyme mixes (Invitrogen). Similar to overexpression cloning, for the target gene RNAi construct, an approximately 150-bp specific sequence of each gene was amplified and cloned into the pDONR-Zeo vector. The resulting positive plasmids were recombined into the pCAMGFP-CvMV-GWi (Graham et al. 2007) binary vector using the Gateway LR Clonase II enzyme mixes (Invitrogen). The resulting plasmids were introduced by electroporation into A. rhizogenes K599 and used for hairy root transformation, as previously described (Libault et al. 2009). Briefly, apical stem sections were excised from 14-day-old soybean seedlings and infected by A. rhizogenes K599 harboring either empty vector as a control or the miRNA precursor expression constructs. After 24 days of incubation, the soybean hairy roots were inoculated with $B$. japonicum USDA110. Transgenic roots were identified 6 weeks postinoculation based on their strong GFP fluorescence. Hairy roots were generated on 12 plants per construct, and the transformation was repeated six times under the same culture conditions. For each replicate, the ratio change of nodule numbers formed on miRNA-expressing roots were compared with control roots transformed with the empty vector and quantified as the relative nodule number of the control $(=1)$. In order to see whether such differences were statistically significant, a $t$ test was performed using the null hypothesis that the fold-change difference between the experimental and control was equal to one.
Acetylene-reduction activity assay.

The acetylene reduction assays were performed essentially as described (Hardy et al. 1968). Briefly, root samples with approximately 5 to 20 nodules were placed into $20-\mathrm{ml}$ glass vials, each fitted with a rubber stoppers. Acetylene $(2 \mathrm{ml})$ was injected into the vial to create a $10 \%$ acetylene atmosphere. These vials were incubated at room temperature. After the given assay time, $2 \mathrm{ml}$ of the head gas from each vial was removed to analyze for ethylene by gas chromatography. Nitrogenase activity was recorded as micromoles of ethylene produced per milligram of nodule fresh weight per hour. The levels of the experimental and control samples were compared with two replications using a $t$ test.

\section{LITERATURE CITED}

Anderson, C. R., Jensen, E. O., Llewellyn, D. J., Dennis, E. S., and Peacock, W. J. 1996. A new hemoglobin gene from soybean: A role for hemoglobin in all plants. Proc. Natl. Acad. Sci. U.S.A. 93:5682-5687.

Appleby, C. A., Tjepkema, J. D., and Trinick, M. J. 1983. Hemoglobin in a nonleguminous plant, parasponia: Possible genetic origin and function in nitrogen fixation. Science 220:951-953.

Aukerman, M. J., and Sakai, H. 2003. Regulation of flowering time and floral organ identity by a MicroRNA and its APETALA2-like target genes. Plant Cell 15:2730-2741.

Broughton, W. J., and Dilworth, M. J. 1971. Control of leghaemoglobin synthesis in snake beans. Biochem. J. 125:1075-1080.

Chen, X. 2004. A microRNA as a translational repressor of APETALA2 in Arabidopsis flower development. Science 303:2022-2025.

Chuck, G., Cigan, A. M., Saeteurn, K., and Hake, S. 2007. The heterochronic maize mutant Corngrass1 results from overexpression of a tandem microRNA. Nat. Genet. 39:544-549.

Cutting, J. A., Schulman, H. M. 1971. The biogenesis of leghemoglobin. The determinant in the Rhizobium-legume symbiosis for leghemoglobin specificity. Biochim. Biophys. Acta 229:58-62.

Cvitanich, C., Pallisgaard, N., Nielsen, K. A., Hansen, A. C., Larsen, K., Pihakaski-Maunsbach, K., Marcker, K. A., and Jensen, E. O. 2000. $C P P 1$, a DNA-binding protein involved in the expression of a soybean leghemoglobin c3 gene. Proc. Natl. Acad. Sci. U.S.A. 97:8163-8168.

Dakora, F. D. 1995. A functional relationship between leghaemoglobin and nitrogenase based on novel measurements of the two proteins in legume root nodules. Ann. Bot. 75:49-54.

De Luis, A., Markmann, K., Cognat, V., Holt, D.B., Charpentier, M., Parniske, M., Stougaard, J., Voinnet, O. 2012. Two microRNAs linked to nodule infection and nitrogen-fixing ability in the legume Lotus japonicus. Plant Physiol. 160:2137-2154.

Graham, T. L., Graham, M. Y., Subramanian, S., and Yu, O. 2007. RNAi silencing of genes for elicitation or biosynthesis of 5-deoxyisoflavonoids suppresses race-specific resistance and hypersensitive cell death in Phytophthora sojae infected tissues. Plant Physiol. 144:728-740.

Hardison, R. C. 1996. A brief history of hemoglobins: Plant, animal, protist, and bacteria. Proc. Natl. Acad. Sci. U.S.A. 93:5675-5679.

Hardy, R. W., Holsten, R. D., Jackson, E. K., and Burns, R. C. 1968. The acetylene-ethylene assay for $\mathrm{n}(2)$ fixation: Laboratory and field evaluation. Plant Physiol. 43:1185-1207.

Ji, L., Liu, X., Yan, J., Wang, W., Yumul, R. E., Kim, Y. J., Dinh, T. T., Liu, J., Cui, X., Zheng, B., Agarwal, M., Liu, C., Cao, X., Tang, G., and Chen, X. 2011. ARGONAUTE10 and ARGONAUTE1 regulate the termination of floral stem cells through two microRNAs in Arabidopsis. PLoS Genet. 7:e1001358. Published online.

Joshi, T., Yan, Z., Libault, M., Jeong, D. H., Park, S., Green, P. J., Sherrier, D. J., Farmer, A., May, G., Meyers, B. C., Xu, D., and Stacey, G. 2010. Prediction of novel miRNAs and associated target genes in Glycine max. BMC Bioinf. 11 (Suppl. 1):S14.

Kereszt, A,, Mergaert, P., and Kondorosi, E. 2011. Bacteroid development in legume nodules: Evolution of mutual benefit or of sacrificial victims? Mol. Plant-Microbe Interact. 24:1300-1309.

Kim, J., Rees, D. C. 1994. Nitrogenase and biological nitrogen fixation. Biochemistry 33:389-397.

Lauter, N., Kampani, A., Carlson, S., Goebel, M., and Moose, S. P. 2005. microRNA172 down-regulates glossy15 to promote vegetative phase change in maize. Proc. Natl. Acad. Sci. U.S.A. 102:9412-9417.

Lelandais-Briere, C., Naya, L., Sallet, E., Calenge, F., Frugier, F., Hartmann, C., Gouzy, J., and Crespi, M. 2009. Genome-wide Medicago truncatula small RNA analysis revealed novel microRNAs and isoforms differentially regulated in roots and nodules. Plant Cell 21:2780-2796.

Li, H., Deng, Y., Wu, T., Subramanian, S., and Yu, O. 2010. Misexpression 
of miR482, miR1512, and miR1515 increases soybean nodulation. Plant Physiol. 153:1759-1770.

Libault, M., Thibivilliers, S., Bilgin, D. D., Radwan, O., Benitez, M., Clough, S. J., and Stacey, G. 2008. Identification of four soybean reference genes for gene expression normalization. Plant Genome 1:44-54.

Libault, M., Joshi, T., Takahashi, K., Hurley-Sommer, A., Puricelli, K. Blake, S., Finger, R. E., Taylor, C. G., Xu, D., Nguyen, H. T., and Stacey, G. 2009. Large-scale analysis of putative soybean regulatory gene expression identifies amyb gene involved in soybean nodule development. Plant Physiol. 151:1207-1220.

Ljones, T., and Burris, R. H. 1972. ATP hydrolysis and electron transfer in the nitrogenase reaction with different combinations of the iron protein and the molybdenum-iron protein. Biochim. Biophys. Acta 275:93-101.

Malik, N. S., Calvert, H. E., and Bauerm, W. D. 1987. Nitrate induced regulation of nodule formation in soybean. Plant Physiol. 84:266-271.

Mathieu, J., Yant, L. J., Murdter, F., Kuttner, F., and Schmid, M. 2009. Repression of flowering by the miR172 target SMZ. PLoS Biol. 7:e1000148. Published online.

Murray, J. D. 2011. Invasion by invitation: Rhizobial infection in legumes. Mol. Plant-Microbe Interact. 24:631-639.

Nair, S. K., Wang, N., Turuspekov, Y., Pourkheirandish, M., Sinsuwongwat, S., Chen, G., Sameri, M., Tagiri, A., Honda, I., Watanabe, Y., Kanamori, H., Wicker, T., Stein, N., Nagamura, Y., Matsumoto, T., and Komatsuda, T. 2010. Cleistogamous flowering in barley arises from the suppression of microRNA-guided $H v A P 2$ mRNA cleavage. Proc. Natl. Acad. Sci. U.S.A. 107:490-495.

O’Brian, M. R., Kirshbom, P. M., and Maier, R. J. 1987. Bacterial heme synthesis is required for expression of the leghemoglobin holoprotein but not the apoprotein in soybean root nodules. Proc. Natl. Acad. Sci. U.S.A. 84:8390-8393.

Ohto, M. A, Fischer, R. L., Goldberg, R. B., Nakamura, K., and Harada, J. J. 2005. Control of seed mass by APETALA2. Proc. Natl. Acad. Sci. U.S.A. 102:3123-3128.

Pessi, G., Ahrens, C. H., Rehrauer, H., Lindemann, A., Hauser, F., Fischer, H. M., and Hennecke, H. 2007. Genome-wide transcript analysis of Bradyrhizobium japonicum bacteroids in soybean root nodules. Mol. Plant-Microbe Interact. 20:1353-1363.

Roth, E., Jeon, K., and Stacey, G. 1988. Homology in endosymbiotic systems: The term "symbiosome". Pages 220-225 in: Molecular Genetics of Plant-Microbe Interactions. R. Palacios and D. P. S. Verma, eds. American Phytopathological Society Press, St. Paul, MN, U.S.A.

Rubio-Somoza, I., and Weigel, D. 2011. MicroRNA networks and developmental plasticity in plants. Trends Plant Sci. 16:258-264.

Shimoda, Y., Shimoda-Sasakura, F., Kucho, K., Kanamori, N., Nagata, M., Suzuki, A., Abe, M., Higashi, S., Uchiumi, T. 2009. Overexpression of class 1 plant hemoglobin genes enhances symbiotic nitrogen fixation activity between Mesorhizobium loti and Lotus japonicus. Plant J. 57:254-263.

Subramanian, S., Hu, X., Lu, G., Odelland, J. T., and Yu, O. 2004. The promoters of two isoflavone synthase genes respond differentially to nodulation and defense signals in transgenic soybean roots. Plant Mol. Biol. 54:623-639.

Valdes-Lopez, O., Yang, S. S., Aparicio-Fabre, R., Graham, P. H., Reyes, J. L., Vance, C. P., and Hernandez, G. 2010. MicroRNA expression profile in common bean (Phaseolus vulgaris) under nutrient deficiency stresses and manganese toxicity. New Phytol. 187:805-818.

Varkonyi-Gasic, E., Wu, R., Wood, M., Walton, E. F., and Hellens, R. P. 2007. Protocol: A highly sensitive RT-PCR method for detection and quantification of microRNAs. Plant Methods 3:12.

Wang, J. W., Park, M. Y., Wang, L. J., Koo, Y., Chen, X. Y., Weigel, D., and Poethig, R. S. 2011. miRNA control of vegetative phase change in trees. PLoS Genet. 7:e1002012. Published online.

Wang, Y., Li, P., Cao, X., Wang, X., Zhang, A., and Li, X. 2009. Identification and expression analysis of miRNAs from nitrogen-fixing soybean nodules. Biochem. Biophys. Res. Commun. 378:799-803.

Wittenberg, J. B., Appleby, C. A., and Wittenberg, B. A. 1972. The kinetics of the reactions of leghemoglobin with oxygen and carbon monoxide. J. Biol. Chem. 247:527-531.

Wu, G., Park, M. Y., Conway, S. R., Wang, J. W., Weigel, D., and Poethig, R. S. 2009. The sequential action of miR156 and miR172 regulates developmental timing in Arabidopsis. Cell 138:750-759.

Wurschum, T., Gross-Hardt, R., and Laux, T, 2006. APETALA2 regulates the stem cell niche in the Arabidopsis shoot meristem. Plant Cell 18:295-307.

Yant, L., Mathieu, J., Dinh, T. T., Ott, F., Lanz, C., Wollmann, H., Chen, X., and Schmid, M. 2010. Orchestration of the floral transition and floral development in Arabidopsis by the bifunctional transcription factor APETALA2. Plant Cell 22:2156-2170.

Zhang, B., Pan, X., Cobb, G. P., and Anderson, T. A. 2006. Plant microRNA: A small regulatory molecule with big impact. Dev. Biol. 289:3-16.

Zhao, Y., Wei, T., Yin, K. Q., Chen, Z., Gu, H., Qu, L. J., and Qin, G. 2012. Arabidopsis RAP2.2 plays an important role in plant resistance to $\mathrm{Bo}$ trytis cinerea and ethylene responses. New Phytol. 195:450-460.

\section{AUTHOR-RECOMMENDED INTERNET RESOURCES}

miRBase, the microRNA database: mirbase.org

The Phytozome database: www.phytozome.net 